\title{
Anisotropic Magnetic Supraparticles with a Magnetic Particle Spectroscopy Fingerprint as Indicators for Cold-Chain Breach
}

\author{
Stephan Müssig ${ }^{1}$, Tim Granath ${ }^{1}$, Tim Schembri ${ }^{1}$, Florian Fidler ${ }^{2}$, Daniel Haddad ${ }^{2}$, Karl-Heinz
} Hiller $^{2}$, Susanne Wintzheimer ${ }^{1}$, Karl Mandel ${ }^{1,3 *}$

1) University of Wuerzburg, Chemical Technology of Materials Synthesis, Roentgenring 11, 97070 Würzburg, Germany

2) Magnetic Resonance and X-ray Imaging Department, Development Center X-ray Technology EZRT, a division of -Fraunhofer Institute for Integrated Circuits IIS, Am Hubland, 97074 Würzburg, Germany

3) Fraunhofer Institute for Silicate Research ISC, Neunerplatz 2, 97082 Würzburg, Germany

Description of magnetic particle spectroscopy (MPS)

In magnetic particle spectroscopy the magnetization behavior of magnetic particles in an alternating sinusoidal magnetic field (Figure S1 a) is measured. The time-dependent magnetization of particles (Figure S1 b) induces a voltage (Figure S1 c). After Fourier-transformation, higher harmonics are observed in the frequency spectrum due to the non-linear magnetic response of ferro-, ferri-, and superparamagnetic particles. The absolute signal intensity of the studied samples is directly proportional to their mass, the amplitude and frequency of the external magnetic field, and the sensitivity of the pick-up coils. To investigate the relative curve behavior, the amplitude intensities are normalized to intensities relative to the fundamental intensity and subsequently analyzed. ${ }^{1}$

a) Applied magnetic field

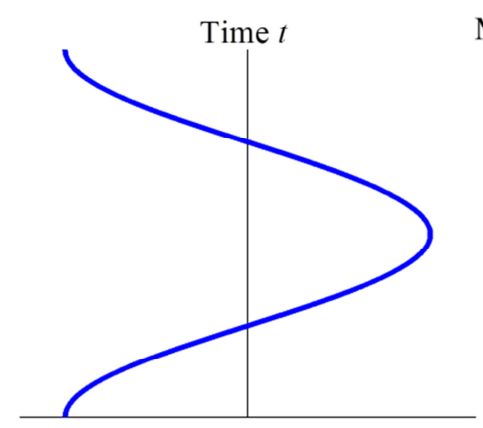

Magnetic field $H$ b) Magnetization

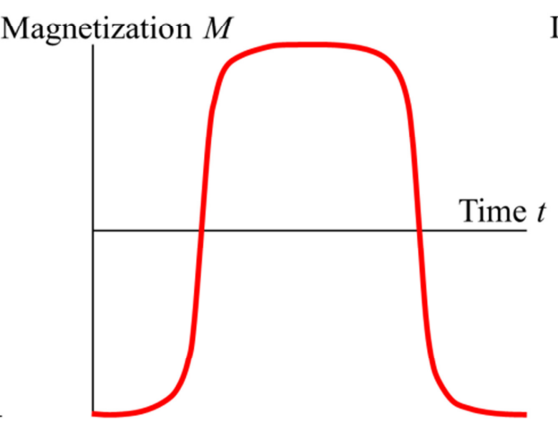

c) Induced voltage

Induced voltage $U$

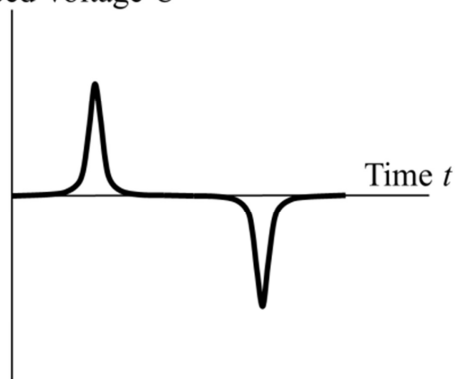

Figure S1. Principle of MPS: When a sinusoidal magnetic field is applied to ferro-, ferri-, or superparamagnetic samples (a), their time-dependent magnetization (b) induces a voltage (c). After Fourier-transformation of the induced voltage, typically the amplitude intensity as a function of higher harmonics is studied. Adapted with permission from Müssig, S.; Fidler, F.; Haddad, D.; Hiller, K.-H.; Wintzheimer, S.; Mandel, K.. Supraparticles with a Magnetic Fingerprint Readable by Magnetic Particle Spectroscopy: An Alternative beyond Optical Tracers. Adv. Mater. Technol. 2019, 291, 1900300. ${ }^{1}$ Copyright 2019 WILEY-VCH Verlag GmbH \& Co. KGaA. 

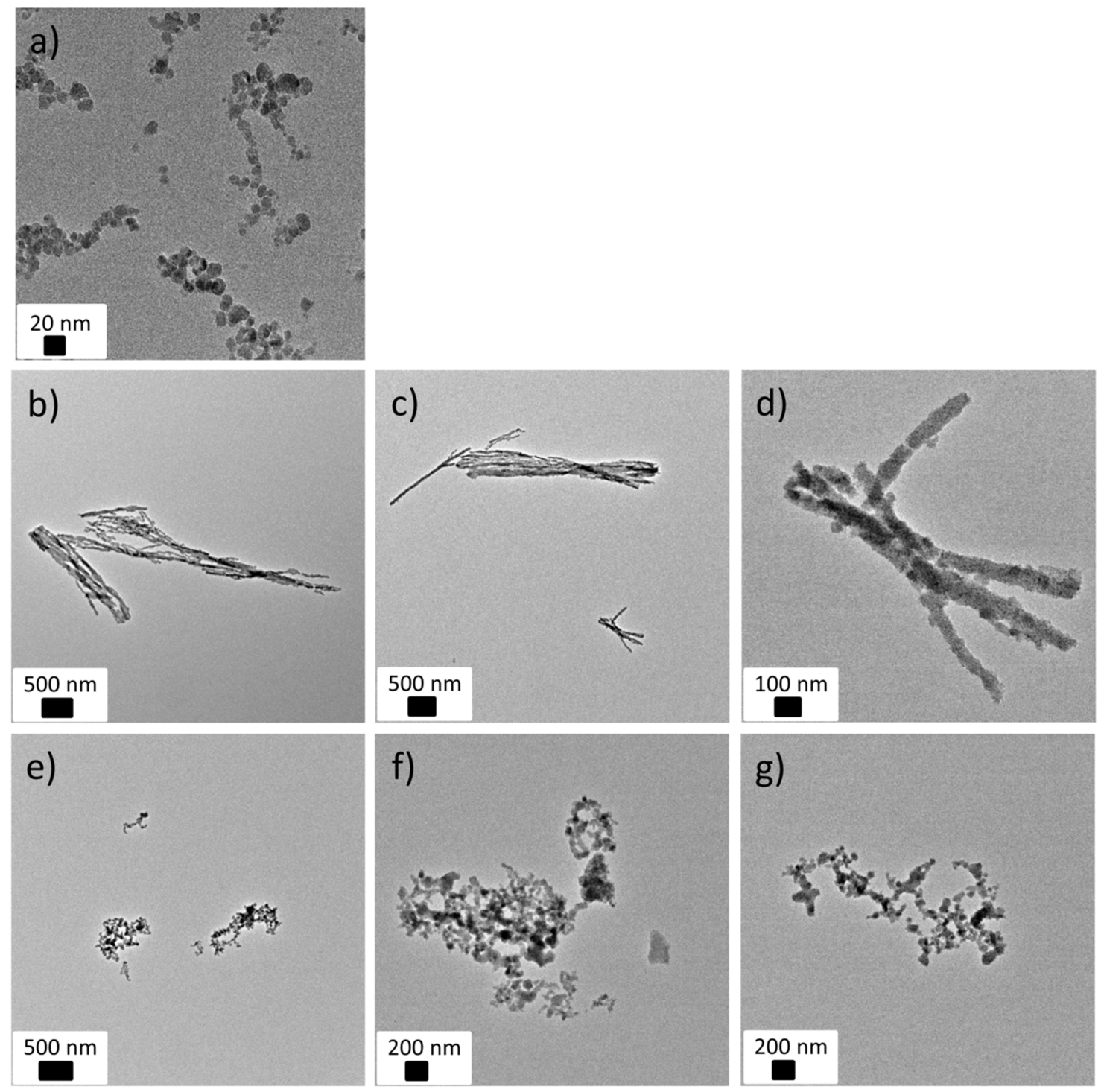

Figure S2. TEM micrograph of iron oxide nanoparticles (a). Adapted with permission from Müssig, S.; Fidler, F.; Haddad, D.; Hiller, K.-H.; Wintzheimer, S.; Mandel, K.. Supraparticles with a Magnetic Fingerprint Readable by Magnetic Particle Spectroscopy: An Alternative beyond Optical Tracers. Adv. Mater. Technol. 2019, 291, 1900300. ${ }^{1}$ Copyright 2019 WILEYVCH Verlag GmbH \& Co. KGaA. TEM micrographs of supraparticles built from the nanoparticles with anisotropic shape (b, c, d) and isotropic morphologies (e, f, g). 


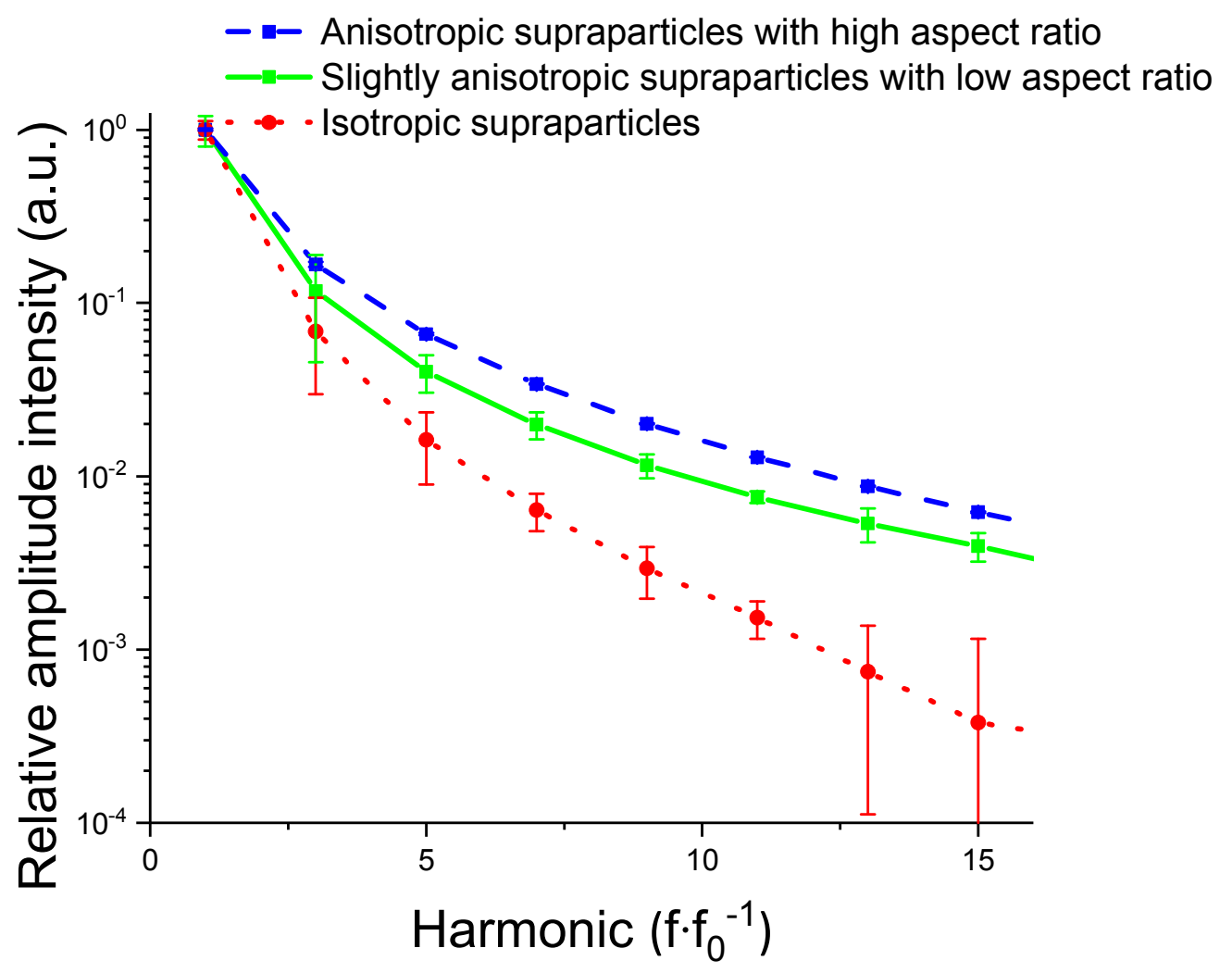

Figure S3: Amplitude intensity normalized to the corresponding fundamental intensity as a function of the higher harmonics of anisotropic supraparticles with high aspect ratio in dispersion (blue dashed line), slightly anisotropic supraparticles with low aspect ratio in dispersion (green solid line) and isotropic supraparticles (red dotted line) measured via MPS with a frequency of $20.1 \mathrm{kHz}$. It becomes clear that the amplitude intensity is enhanced with a more pronounced anisotropic structure. The only slightly anisotropic supraparticles with low aspect ratio were synthesized as described in a recent work ${ }^{2}$ by decreasing the magnetic field strength during assembly of the iron oxide nanoparticles to approximately $15 \mathrm{mT}$.
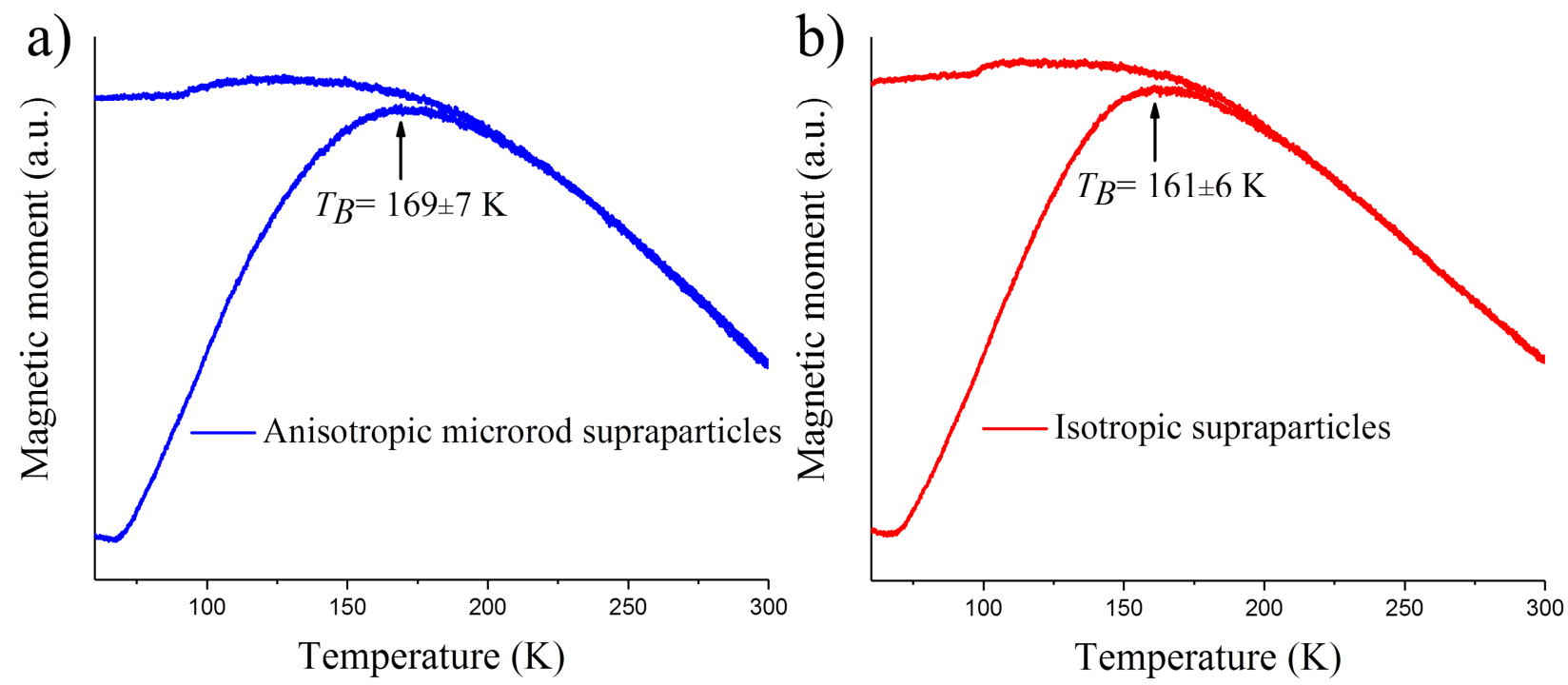

Figure S4. Zero-field-cooled field-cooled measurements of anisotropic microrod (a) and isotropic (b) supraparticles with their respective blocking temperature $T_{\mathrm{B}}$. The similar signal curves indicate that it is not a change in inter-particle interactions which is responsible for the altered magnetic properties. 


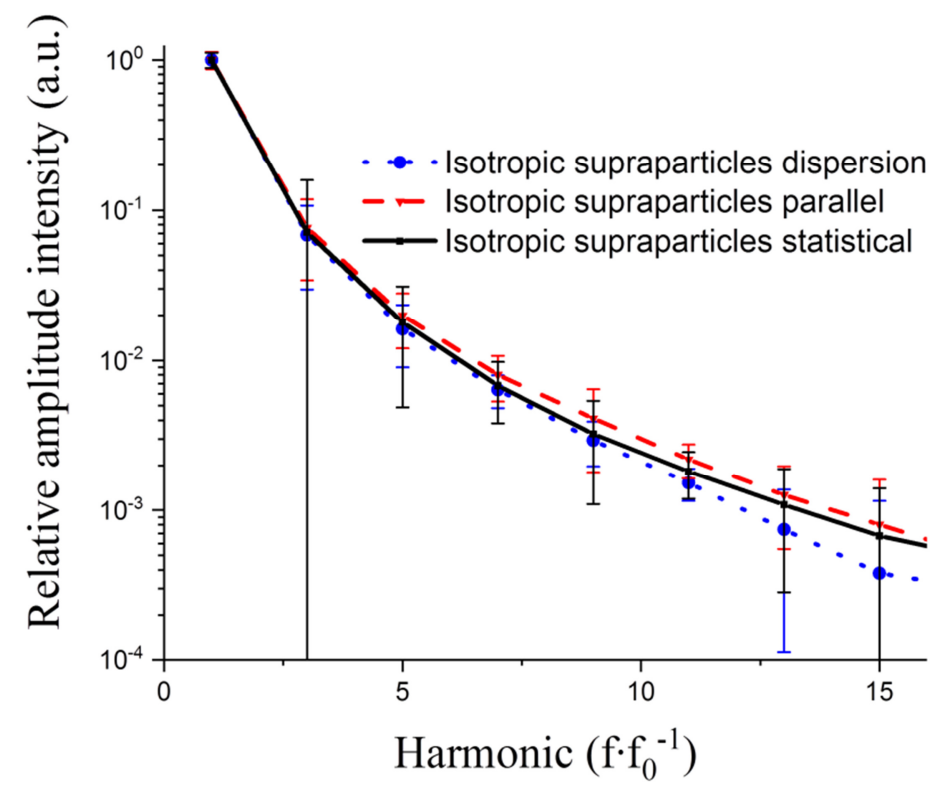

Figure S5. Amplitude intensity normalized to the corresponding fundamental intensity as a function of the higher harmonics of isotropic supraparticles in dispersion (blue dotted line), and oriented in parallel (red dashed line) or statistically (black solid line) towards an external magnetic field measured via MPS with a frequency of $20.1 \mathrm{kHz}$. Signal variations smaller than the standard deviation indicate negligible anisotropy.
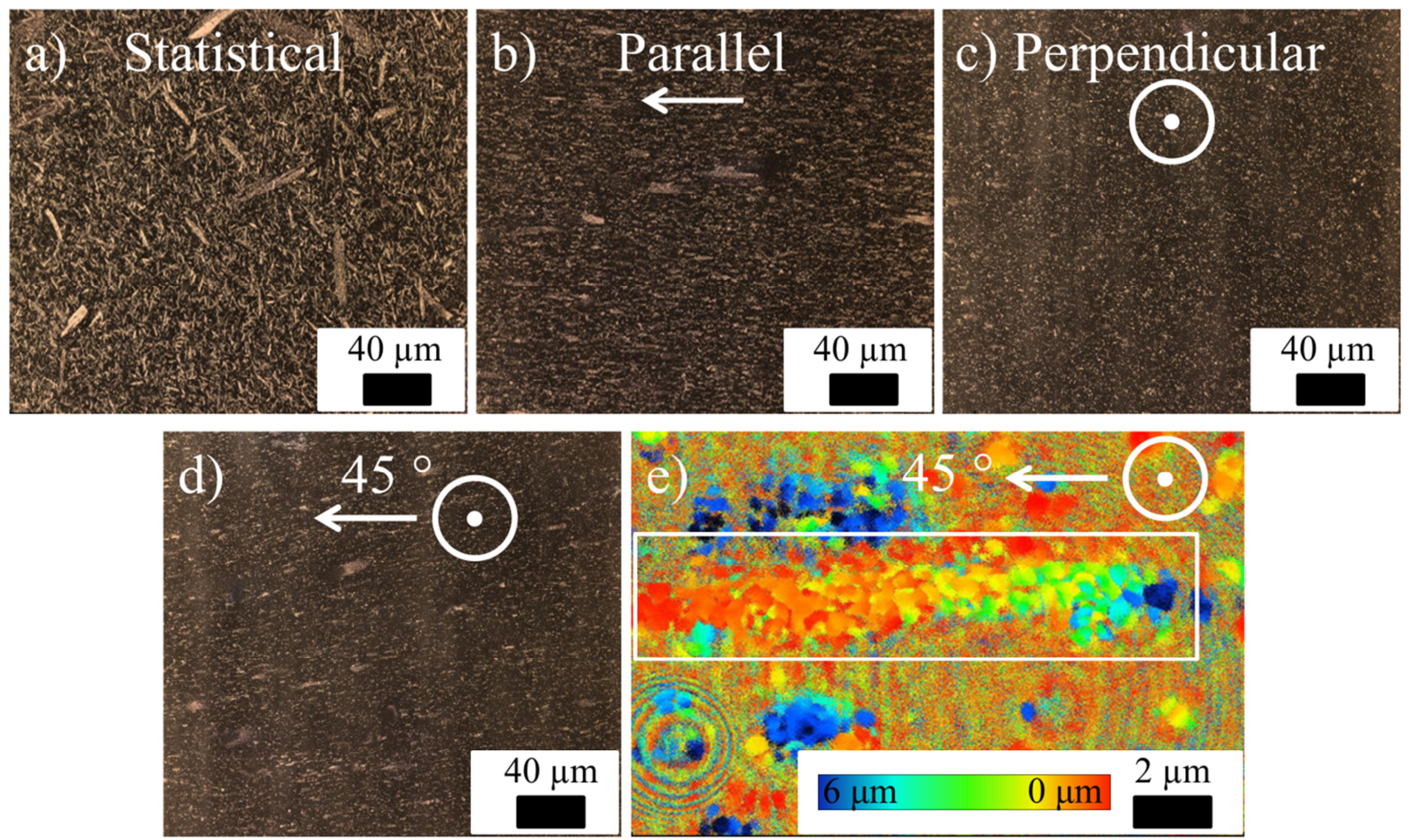

Figure S6. LSM images of TMPTA: TMPTA-E 2:8 foils with differently oriented anisotropic microrod supraparticles towards an external field: No magnetic field yields a statistical orientation (a), whereas application of a magnetic field parallel (b) or perpendicular (c) to the lateral expansion of the foil leads to preferential orientations of the microrod supraparticles. A $45^{\circ}$ orientation (d) towards an external magnetic field can barely be distinguished to a parallel orientation with a single image, but can be confirmed by the diagonal altitude profile of individual microrod supraparticles (e, white frame). 


\section{Experimental details}

Superparamagnetic anisotropic microrods were obtained by a two-step synthesis as described elsewhere. ${ }^{2}$ Firstly, iron oxide nanoparticles were obtained in a continuous precipitation process by dissolving $\mathrm{FeCl}_{3} \cdot 6 \mathrm{H}_{2} \mathrm{O}(10.80 \mathrm{~g}, 40 \mathrm{mmol}$, Sigma Aldrich, $>99 \%)$ and $\mathrm{FeCl}_{3} \cdot 4 \mathrm{H}_{2} \mathrm{O}(3.98 \mathrm{~g}, 20 \mathrm{mmol}$, Fluka, $>99 \%)$ in deionized water $(125 \mathrm{~mL})$ at room temperature and mixing it with $5 \mathrm{wt} \%$ aqueous ammonia solution $\mathrm{NH}_{3}$ (aq.) $(125 \mathrm{~mL}$ ) by pumping both fluids with a peristaltic pump (Ismatec MCP, flow rate: $1000 \mathrm{~mL} \cdot \mathrm{min}^{-1}$ ) through a Y-piece. Subsequently, the black precipitate was magnetically separated after $60 \mathrm{~s}$, washed three times with deionized water and redispersed in deionized water $(250 \mathrm{~mL})$ for 5 min. To obtain a stable ferrofluid, $1 \mathrm{M}$ nitric acid $(50 \mathrm{~mL})$ was added while stirring for $5 \mathrm{~min}$ before deionized water $(250 \mathrm{~mL})$ was added and stirred for $18 \mathrm{~h}$.

Secondly, anisotropic superstructures were synthesized by dropwise addition of $1.2 \mathrm{~mL}$ tetraethoxyorthosilicate (TEOS) into $24 \mathrm{~mL}$ of the as-prepared ferrofluid and overlaid with $10 \mathrm{~mL}$ cyclohexane. Then, $125 \mathrm{~mL}$ acetone was quickly poured into the mixture while the whole system was exposed to a magnetic field $(>120 \mathrm{mT})$ leading to anisotropic microrod supraparticles with a high aspect ratio. For the synthesis of slightly anisotropic supraparticles with a low aspect ratio and isotropic supraparticles, a magnetic field of $\sim 15 \mathrm{mT}$ and $0 \mathrm{mT}$, respectively, was applied while the rest of the synthesis was kept unchanged. Finally, the precipitate was magnetically separated, decanted and washed with $0.1 \mathrm{M} \mathrm{NaOH}(0.3 \mathrm{~mL})$ in acetone $(125 \mathrm{~mL})$. The mixture was further washed twice with a mass weighted 2:1 acetone-water mixture $(180 \mathrm{~mL})$, and twice with deionized water $(180 \mathrm{~mL})$. The washed precipitate was redispersed in EtOH $(15 \mathrm{~mL})$.

\section{Transmission electron microscopy (TEM)}

Transmission electron micrographs were acquired using a JEM-2010 TEM from JEOL Ltd., Akishima, Japan at $200 \mathrm{kV}$ acceleration voltage.

\section{Immobilization of microrod supraparticles}

For immobilization in acrylate, the as-prepared $1.5 \mathrm{wt}-\%$ microrod supraparticle dispersion in EtOH was utilized. A 2:8 mixture by weight of trimethylolpropane triacrylate (TMPTA) and ethoxylated (20) trimethylolpropane triacrylate (TMPTA-E), respectively, containing $2.0 \mathrm{wt}-\%$ of an IRGACURE 184 photoinitiator was used as the acrylate monomer solution. The dispersion and acrylate solution were mixed in a mass weighted 1:2 ratio. The EtOH solvent was evaporated at $80{ }^{\circ} \mathrm{C}$. At room temperature the dispersion was mixed for $10 \mathrm{~s}$ at $3000 \mathrm{rpm}$ on a vortex mixer before applying $0.05 \mathrm{~mL}$ into a borosilicate glass tube with $6 \mathrm{~mm}$ in diameter. UV curing was conducted using a bluepoint $3 \mathrm{UV}$-spotlight from Dr. Hönle, Gräfelfing, Germany, irradiating the substrate for $120 \mathrm{~s}$. Simultaneous magnetic orientation of the microrod supraparticles was performed by placing a ferromagnet (Q-51-51-25-N, supermagnete.de) at a distance from magnet surface to glass tube surface of $13 \mathrm{~cm}$ to the substrate and exposing the foil to the magnetic field of approximately $5 \mathrm{mT}$ for $5 \mathrm{~s}$ before irradiation. By shifting the magnet perpendicular to the glass, parallel to the substrate, and at a $45^{\circ}$ angle for an orientation in $45^{\circ}$, different orientations in the glass tube were achieved. The glass tubes were used as prepared for MPS measurements.

To freeze the microrod supraparticles in water for the proof-of-concept experiment, liquid nitrogen was poured onto $6 \mathrm{~mm}$ glass tubes containing the microrod supraparticles dispersed in water $(50 \mu \mathrm{L})$ while magnetically orienting them as described. After MPS measurement, the dispersions were defrosted at room temperature for approximately 30 min before the next MPS measurement was conducted at a few degree Celsius sample temperature with subsequent refreezing with liquid nitrogen.

\section{Magnetic measurements}

Magnetization measurements were performed at $300 \mathrm{~K}$ with a vibrating sample magnetometer VersaLabTM $3 T$ from Quantum Design, San Diego, USA in a magnetic field range of $\pm 30,000$ Oe. The measurement speed was set to 5 Oe $\cdot \mathrm{s}^{-1}$ between $\pm 5,000$ Oe and $50 \mathrm{Oe} \cdot \mathrm{s}^{-1}$ exteriorly. For zero-field-cooled field-cooled (ZFC/FC) measurements, a VersaLabTM $3 T$ from Quantum Design was used and samples were cooled to $60 \mathrm{~K}$ without external field before heating to $300 \mathrm{~K}$ and subsequent cooling to $60 \mathrm{~K}$ with $12 \mathrm{~K} \cdot \mathrm{min}^{-1}$ with a field of $10 \mathrm{Oe}$.

The magnetic properties of the embedded or mobile microrod supraparticles were characterized with a MPS unit, Pure Devices $\mathrm{GmbH}$, Rimpar, Germany, in a sinusoidal alternating field of $20.1 \mathrm{kHz}$ from -300 Oe to 300 Oe. These parameters were chosen because $20 \mathrm{kHz}$ seems to be the most commonly used frequency in literature and the amplitude intensity of \pm 300 Oe provides as much information as possible about the investigated samples with the employed MPS machine. Measurements were performed at room temperature, while the frozen samples for cold chain detection were colder than $0{ }^{\circ} \mathrm{C}$.

Laser scanning microscopy (LSM)

Verification of the three different orientations of microrod supraparticles in the acrylate foils was conducted using confocal laser scanning microscopy (LSM) with a Keyence color $3 D$ VK-X210 laser scanning microscope.

\section{REFERENCES}

(1) Müssig, S.; Fidler, F.; Haddad, D.; Hiller, K.-H.; Wintzheimer, S.; Mandel, K. Supraparticles with a Magnetic Fingerprint Readable by Magnetic Particle Spectroscopy: An Alternative beyond Optical Tracers. Adv. Mater. Technol. 2019, $291,1900300$.

(2) Mandel, K.; Granath, T.; Wehner, T.; Rey, M.; Stracke, W.; Vogel, N.; Sextl, G.; Müller-Buschbaum, K. Smart Optical Composite Materials: Dispersions of Metal-Organic Framework@Superparamagnetic Microrods for Switchable IsotropicAnisotropic Optical Properties. ACS nano 2017, 11, 779-787. 